Textures and Microstructures, 1988, Vols. 8 \& 9, pp. 509-529

Reprints available directly from the publisher

Photocopying permitted by license only

(C) 1988 Gordon and Breach Science Publishers Inc.

Printed in the United Kingdom

\title{
The Sphere Rotation Method: Determination of Low Energy Orientation Relationships by an $X-R a y$ Texture Analysis
}

\section{R. MAURER}

Max-Planck-Institut für Metallforschung, Institut für Werkstoffwissenschaften, D-7000 Stuttgart, FRG

(Received July 15, 1987)

\section{Dedicated to the memory of Professor Günter Wassermann}

Sphere rotation experiments are reviewed, with emphasis on the latest results. The method and its evaluation are described in detail. The sphere rotation method has been applied so far to study the correlation between structure and energy of homoand heterophase boundaries, also to study the relation of temperature, pressure, mobility, corrosivity, and embrittlement to the energy of grain (=homophase) boundaries. The material specific properties, expecially the electronic structure, influence the number of low energy orientations to a great extent. All current criteria developed for the explanation of low energy boundary orientations do not fit the observations in a unique way. The common characteristics of hetero- and homophase boundaries are represented.

KEY WORDS: Grain boundaries, phase boundaries, low energy boundaries, sphere rotation method, pole figure measurements, corrosion, embrittlement, electronic structure.

\section{INTRODUCTION}

In the past ten years the sphere rotation method has proven to be a suitable means of determining low energy orientation relationships of phase and grain boundaries as a function of many parameters (such as pressure, temperature, bonding type etc.). These investigations have led to a fundamental understanding of the behaviour of 
such boundaries. In the present study, measurements of this kind are presented and discussed critically. In this contribution the latest results will be emphasized, especially those describing the influence of the electronic structure on the behaviour of boundaries

\section{METHOD}

The sphere rotation effect was first suggested by Shewmon (1966) for diffusion measurements. When two single crystalline spheres are sintered together, an interface between both spheres is formed. Comparable diffusion processes develop by sintering a sphere on a plate (Figure 1a). The interfacial energy depends on the orientation relationships between the two crystals (Figure 1b). By annealing the specimen, the two crystals rotate relative to one another into an orientation of low energy. In this position, the sphere has achieved a state of local equilibrium. In this form, the technique was not easy to apply because it required the measurement of many orientation relationships. Herrmann et al. (1976) opened the way for utilizing the sphere rotation effect by using a large number of spheres in contact with a flat single crystal plate (Figure 2). This kind of experiment is known as sphere-rotation method or sphere/plate method or rotating-sphere-on-a-plate method. When two spheres

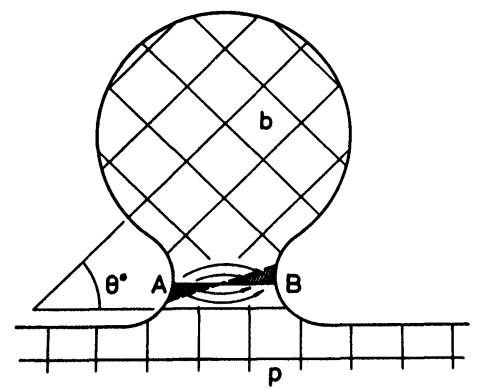

Figure 1a Schematic diagram showing a boundary (AB) formed by sintering a single crystal ball (b) onto a mono crystal plate (p). The crystal lattices of the ball and the plate are misorientated with respect to one another by an angle $\theta^{*}$. The atomic flux indicated by the arrows results in an anti-clockwise rotation of the sphere so that the misorientation angle changes from $\theta^{*}$ to $\theta_{0}$ (Fig. 1b) (from Herrmann et al., 1976). 


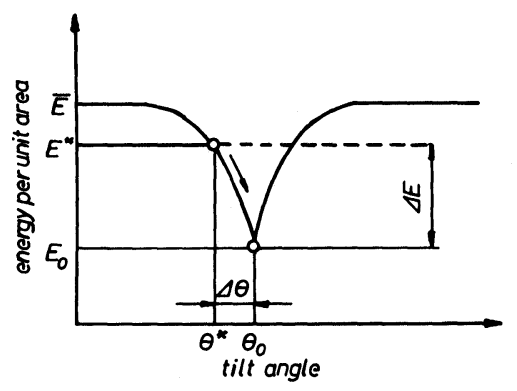

Figure 1b Schematic curve of the grain boundary energy per unit area versus tilt angle. A boundary of low energy is obtained for the orientation relationship corresponding to the tilt angle $\theta_{0}$ (from Herrmann et al., 1976).

relative to one another are moved, all five degrees of freedom of the boundary are changed. In contrast to this, a fixed plate restricts the sphere rotation to three degrees of freedom. Despite this restriction, the advantages of measurement are prevailing.

At the beginning of the annealing time, a statistical distribution of orientations of the spheres on the plate can be observed. During subsequent annealing, the spheres rotate and, after the rotation has finished, the total spectrum of low energy orientation relationships can be measured (Figure 3). X-ray texture analysis was used to determine the distribution of the orientations of the spheres. To a first approximation, the intensity of the diffracted X-ray reflections

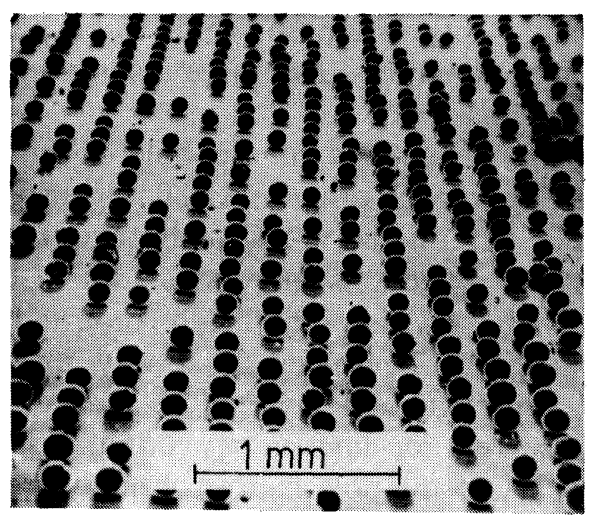

Figure 2a Scanning electron micrograph showing the lateral arrangement of the spheres on the plate (from Herrmann et al., 1976). 


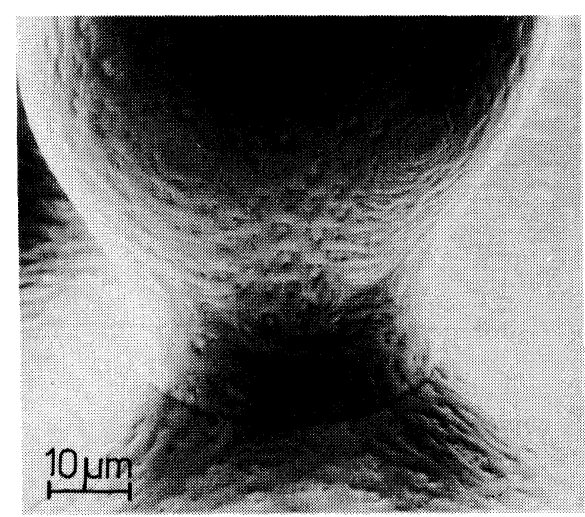

Figure 2b Scanning electron micrograph of a sphere sintered onto a monocrystal plate (from Herrmann et al., 1976).

is proportional to the number of spheres rotated into an orientation relationship of low energy and thus, proportional to the width (catchment area) of the corresponding energy cusp (Goodhew and Allen, 1977). For grain boundaries it can be assumed that the width of a cusp is nearly proportional to its depth (in a boundary energy vs. lattice misorientation plot) (Goodhew, 1980). This is correct if the shapes of all cusps are such that they have equal slopes. Since the slope is determined by the number of dislocations which have to be introduced into the boundary in order to create a given misorientation increment, the above mentioned proportionality between width and depth of a cusp seems to be justified according to a first rough approximation. The same assumption should also be valid for phase boundaries.

If the number of reflections in the pole figure is small, those reflections belonging to each other and defining a special orientation relationship can be determined in a straight forward manner. This is no longer true if the reflections superpose and/or if more than one reflection possess the crystallographically required angles. Individual reflections can be combined in a way producing fictitious misorientations. Pole figures which can be indexed unambiguously are generally observed for low energy orientations of interphase boundaries, contrary to the pole figures of many grain boundaries.

Maurer (1987) used $\mathrm{Cu}$ spheres on a $\mathrm{Cu}$ plate to show that pole figures with these complications can be evaluated. It was taken into 

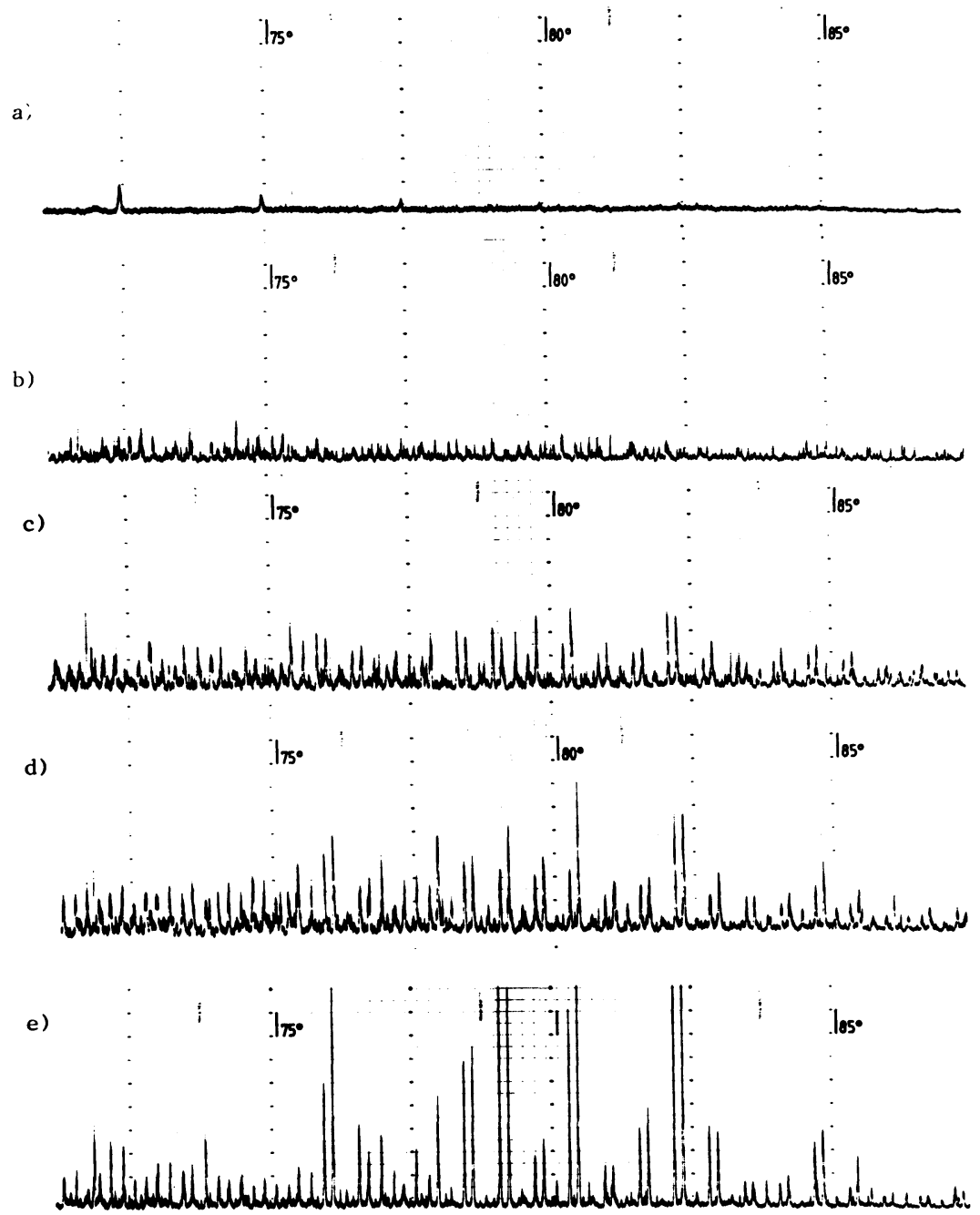

Figure 3 Sections through the $\{111\}$ pole figure of a $\mathrm{Cu} / \mathrm{Cu}$ specimen as a function of the annealing time. (a) a (100) plate without spheres; (b) a specimen annealed at $1030^{\circ} \mathrm{C}$ for $15 \mathrm{~min}$ (random orientations); (c) for $6 \mathrm{~h}$, (d) for $12 \mathrm{~h}$, and (e) for $48 \mathrm{~h}$ (finished rotation). 
consideration that some reflections are superimposed. The ambiguity of overlapping reflections was reduced by evaluating the intensity of the reflections. The orientation distribution was determined by analysing the measured X-ray $\{111\}$ pole figures $\left(\mathrm{Cu}-\mathrm{K}_{\alpha^{-}}\right.$ radiation). This analysis involved calculating the pole figure for various assumed preferred misorientations and comparing the results with the measured pole figure. This process was continued until reasonable, self-consistent results were obtained. By this technique, a number of boundaries were detected which were only weakly represented in the pole figure. Nevertheless, the method for the unequivocal evaluation of these complex pole figures remains to be improved. An approximate method of tackling this problem is to evaluate the highest intensities of the pole figures only (Lojkowski et al., 1987). With this simplification, however, it is possible to disregard important low energy orientations.

Recently, studies have been in progress to apply modern method of texture analysis (ODF-method) to the problem (Bunge and Gleiter, 1987).

\section{GRAIN BOUNDARIES}

Herrmann et al. (1976) used this method for the first time to investigate the orientation relationships of grain boundaries of low energy in $\mathrm{Ag}$ and $\mathrm{Cu}$, respectively. Both materials were found to exhibit different types of low energy boundaries. This result was interpreted in terms of different electronic structures. $\mathrm{Ag}$ and $\mathrm{Cu}$ possess the same crystallographic structure but a different band structure. The particular low energy orientation relationships, identical in both systems, were defined as electronic-insensitive grain boundaries. The other ones, being system specific, were called electronic-sensitive grain boundaries.

Sautter et al. (1977) extend this interpretation to the grain boundaries of $\mathrm{Ag}-\mathrm{Au}$ and $\mathrm{Ag}-\mathrm{Bi}$ alloys. A later study by Maurer and Gleiter (1985) deals with the degree of influence of the electronic structure on the number of low energy orientation relationships in $\mathrm{Ni}-\mathrm{Cu}$ alloys. The results of these investigations will be discussed more extensively in a separate section.

The evaluation of the pole figures obtained by means of sphere- 


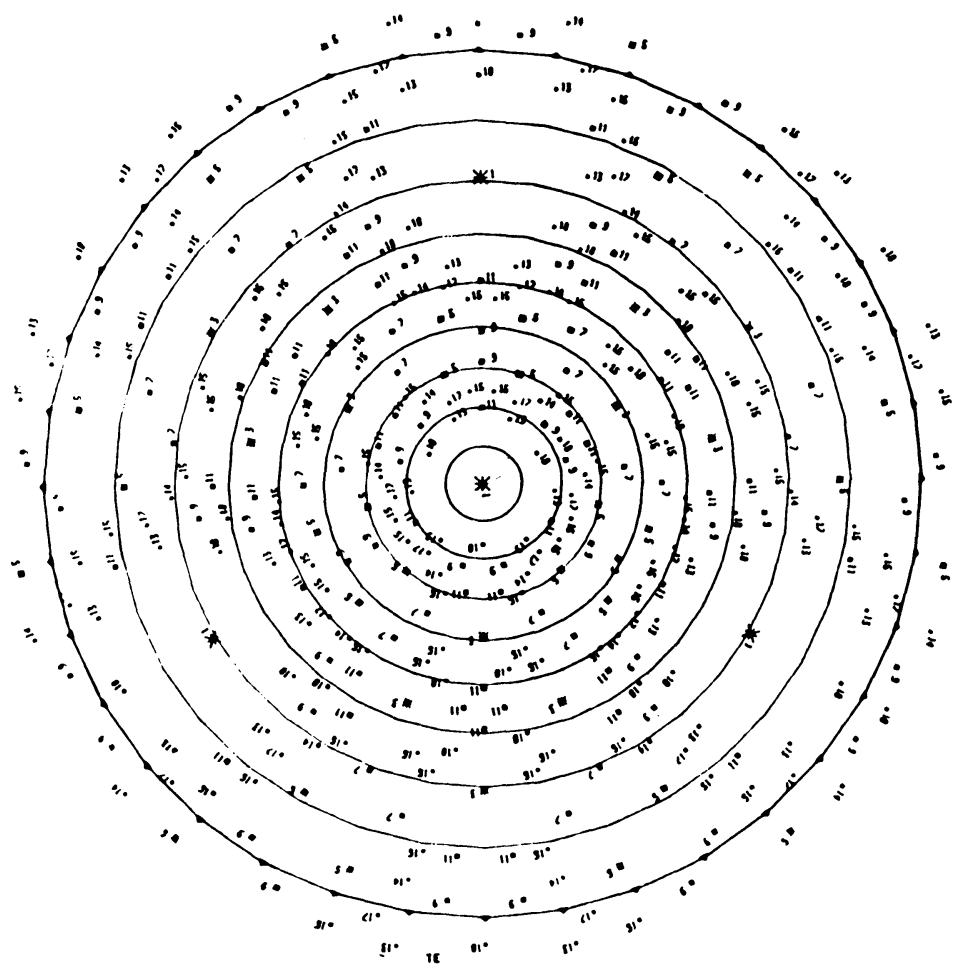

Figure $4\{111\}$ Pole figure of the peak positions theoretically expected by the coincidence model for a (111) orientated plate (from Maurer and Gleiter, 1986).

rotation experiments suggests that a coincidence orientation relationship is not necessarily a prerequisite to low energy orientations (Lojkowski and Gleiter, 1985; Maurer and Gleiter, 1986).

Figure 4 is an example for a calculated pole figure assuming that the energy geometrically depends on the 3-dimensional density of coincidence sites (after data from Mykura, (1980)). Figure 5 shows an experimentally observed pole figure. A comparison reveals that the observed number of low energy orientations is smaller than expected. Based on the detailed evaluation of data of rotated $\mathrm{Cu}$ spheres on a (100) Cu plate (Maurer, 1987), Sutton and Balluffi (1987) pointed out that there exists no obvious correlation between measured orientation relationships of low energy boundaries and current geometric criteria which have been proposed in the litera- 


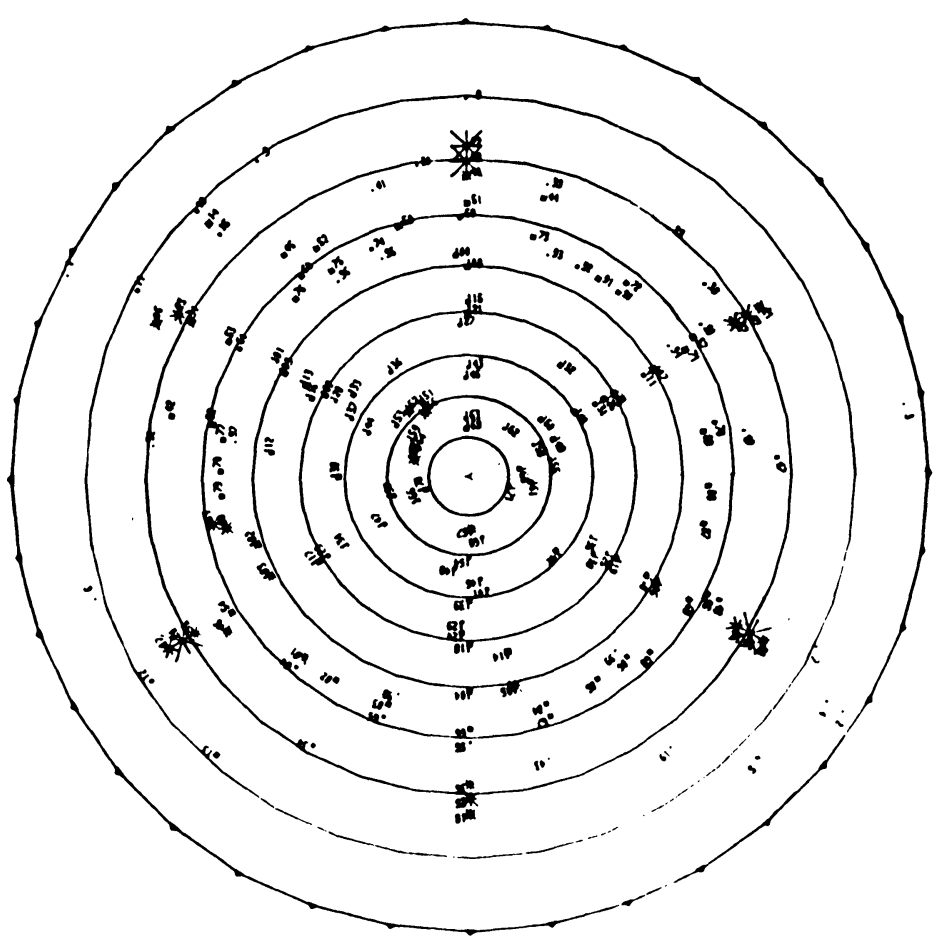

Figure $5\{111\}$ Pole figure of the peak positions measured for a (111) Ag-plate with $\mathrm{Ag}$ spheres annealed at $950^{\circ}$ for $450 \mathrm{~h}$ (from Maurer and Gleiter, 1986).

ture as coincidence site density, grain boundary periodicity, or 0-lattice site density.

The authors confirmed this observation in discussing geometric criteria and comparing them with available experimental results indicating boundaries of relatively low energy.

The low energy boundaries identified by Lojkowski et al. (1987) in $\mathrm{Ag}$ and $\mathrm{Cu}$ can be interpreted on the basis that they form facets. The facets contain densely packed structural units. However, little correlation was found between these structural units and simple existing geometric criteria for low energy. These structural units are composed of symmetrical tilt boundaries of short periodicity or are created by joining together a $\{111\}$ surface with a stepped $\{100\}$ surface in such a way that the ledges on the surface of one grain are 
locked in the surface of the other grain. The effect of the crystallographic orientation of the grain boundary plane on the grain boundary energy is as important as the effect of the misorientation angle. As only the highest intensities are taken into account, no difference was observed as far as the crystallographic parameters of low energy boundaries in copper, silver and a $\mathrm{Cu}$ $0.1 \% \mathrm{Bi}$ alloy at temperatures above $0.95 T_{m}$ are concerned.

As expected from Gibbs' equation, $G=H+p V-T S$, the free energy of a boundary depends on temperature and pressure. Hence, the low energy orientation relationships should be pressure- and temperature-sensitive. In fact, investigations of low energy boundaries in Ag by Meiser and Gleiter (1980) indicate that some special orientations existing at a high pressure were not observed at ambient pressure and vice versa (Figure 6). Also, using the sphere rotation method, Erb and Gleiter (1979) proved that the number of low energy orientations decreases as the boundary entropy is enhanced due to an increase in temperature (Figure 7).

In a study by Lojkowski et al. (1987), low energy orientation

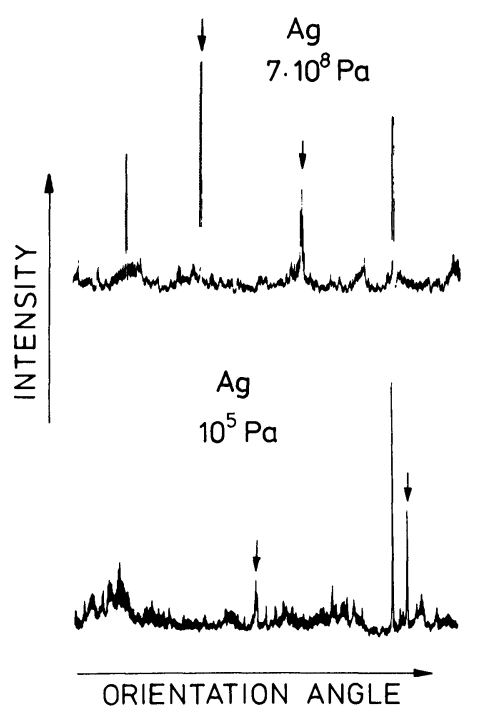

Figure 6 Orientations of low energy in silver at different pressures. The orientation relationships that are dependent on pressure are marked by arrows (from Meiser and Gleiter, 1980). 


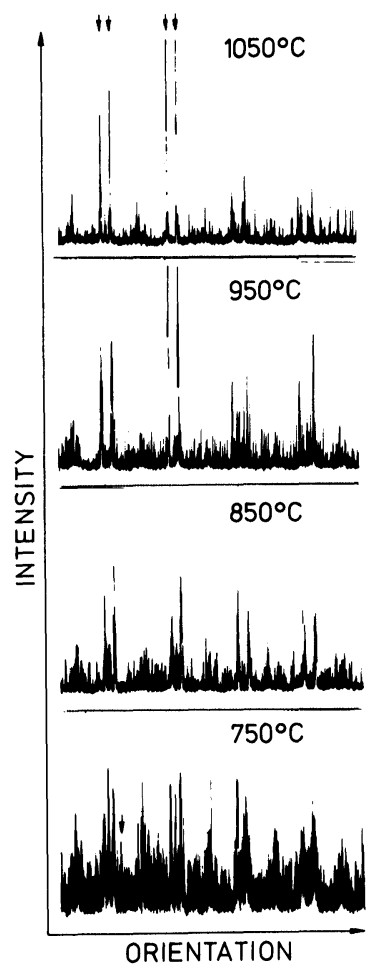

Figure 7 Orientations of the low energy boundaries in copper as a function of the temperature. Some of the special orientation relationships are independent of the temperature. Four of those are marked by arrows in the section at $1050^{\circ} \mathrm{C}$. The peak marked in the recording at $750^{\circ} \mathrm{C}$ is not present at $1050^{\circ} \mathrm{C}$ (from Erb and Gleiter, 1979).

relationships were analysed in detail for $\mathrm{Cu}$ and $\mathrm{Ag}$. At temperatures close to the melting point $\left(0.8-0.996 T_{m}\right),[110]$ tilt boundaries were identified as being associated with those energy minima which exhibited the largest catchment areas for rotating spheres independent of the plate orientation. Within the resolving power, up to $0.8 T_{m}$, no preferred tilt angles of the [110] tilt boundaries can be detected. At higher temperatures, a few special tilt angles are favoured.

If the sphere-rotation method is slightly modified, other properties such as the boundary mobility, corrosivity and boundary 
embrittlement can be investigated and correlated with the boundary structure. For example, Wirth and Gleiter (1981) studied the dependence of boundary mobility on boundary energy in the system $\mathrm{Cu}-5$ at. \% Ag.

The migration of boundaries was caused by the discontinuous precipitation reaction of the supersaturated solid solution. With slight undercooling, discontinuous precipitation is not initiated at low energy boundaries. With increasing undercooling, a growing fraction of low energy boundaries is able to become a discontinuous reaction front. During the discontinuous precipitation reaction, the diffusion coefficient in the interfaces is enhanced by a factor of about $10^{3}$ (consistent with data reported in the literature).

Therefore, it is assumed that a static grain boundary and a discontinuous reaction front have different atomic structures created by a structural transformation.

A further field of research of the sphere rotation method comprises investigations concerning the dependence of the corrosion sensitivity of the interfaces on the boundary misorientations (Erb et al., 1982). These studies were carried out by putting the specimen into a solution which preferably corroded the grain

a)
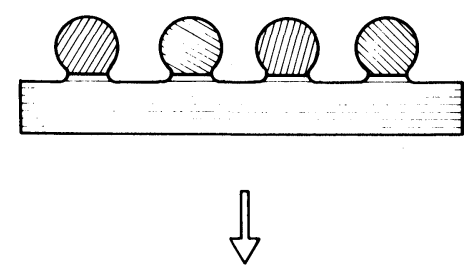

b)

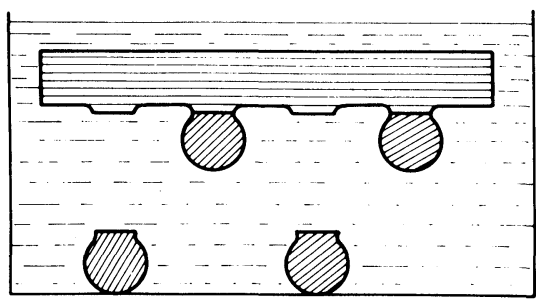

Figure 8 (a) Schematic diagram of the specimens used for the corrosion experiments and (b) the process of removing individual spheres by boundary corrosion (from Erb et al., 1982). 


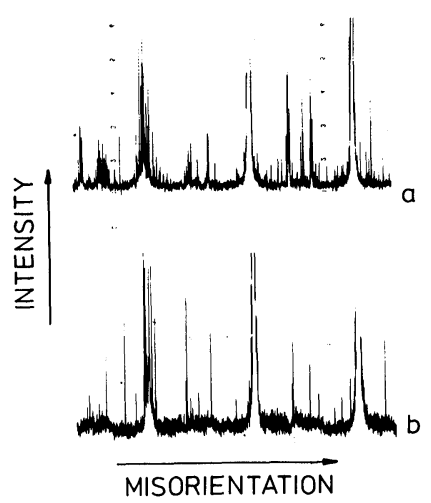

Figure 9 Comparison between identical sections of the $\{111\}$ texture diagrams of two specimens. The specimen of Figure $9 \mathrm{a}$ was annealed for $50 \mathrm{~h}$ at $1050^{\circ} \mathrm{C}$ (without any corrosion) to rotate all the spheres into the orientation relationships of minimum energy. The specimen of Fig. 9b contained randomly oriented spheres and was corroded for $40 \mathrm{~h}$ (from Erb et al., 1982).

boundaries (Figure 8). If the entire boundary region between a sphere and the plate is dissolved, the corresponding sphere falls off. Those spheres whose boundaries have the highest (lowest) corrosion sensitivity are the first (last) to fall off. The orientation relationships of those spheres with the highest and the lowest corrosion sensitivity were determined by texture analysis. The pole figures obtained were compared with pole figures corresponding to boundaries of low energy (sphere rotation experiments) (Figure 9). In the case of grain boundaries in $\mathrm{Cu}$, the comparison of the two pole figures led to the conclusion that boundaries of low energy are least sensitive to corrosion. In the texture diagrams, the reflections of the spheres after corrosion (without rotation) are sharper than those after rotation (without corrosion). This difference is due to the fact that it is not the energy of the grain boundaries per se which defines the corrosion sensitivity in the boundaries but the dislocation structure in the boundary. This was confirmed by microscopic investigations.

Maurer et al. (1984) observed no enhanced corrosion in the crystal lattice in the vicinity of grain boundaries under conditions where the corrosive medium was not in contact with the actual grain boundary. For this experiment, a sphere rotation specimen was used (Figure 10): Plate 1 with the spheres 2 was coated from the 


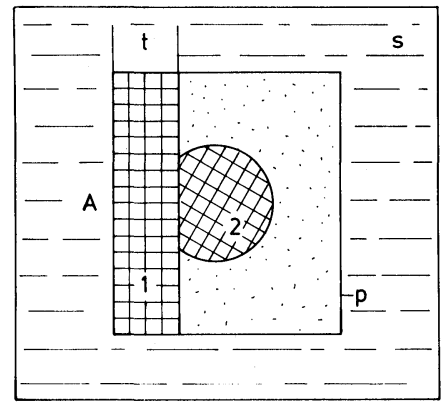

Figure 10 Schematic cross section through the experimental arrangement used in the work reported by Maurer et al. (1984): 1, single-crystal plate; 2, single-crystal sphere; $p$, corrosion protective layer; $s$, corrosive aqueous solution; $A$, corroded area; $t$, thickness of crystal 1 .

"boundary side" with a corrosion protective layer, $p$, and was subsequently immersed into a suitable aqueous solution which was known to result in intergranular corrosion. No selective corrosion in the area, $A$, at the "other side" of a grain boundary was observed irrespective of the alloy composition, the boundary structure, the cooling rate, and the distance, $t$, of the boundary beneath the free surface exposed to the corrosion medium. The minimum spacing, $t^{\prime}$, applied was less than $1 \mu \mathrm{m}$. The experimentally observed width, $d$, of the boundary region which is dissolved selectively during intergranular corrosion is of the order of $20-45 \mu \mathrm{m}$ and hence much larger than the minimum spacing, $t^{\prime}$. Therefore, it was concluded that the width of the intergranular corrosion region is not due to an enhancement of the free energy of the lattice region in the vicinity of a grain boundary. The direct contact between the corrosive agent and the core of a boundary seems to be required to initiate intergranular corrosion.

In an experiment similar to that of Erb and Gleiter (1982), Roy et al. (1982) investigated the embrittlement of the grain boundaries by solute segregation to the boundaries. Those spheres breaking in a brittle manner along the grain boundaries fall off when the plate with spheres is vibrated ultrasonically. Low energy boundaries were found to exhibit the lowest brittleness. These results were interpreted in terms of preferred solute segregation.

This interpretation was confirmed by measurements of the oxygen 
segregation at grain boundaries in $\mathrm{Ag}$ (Schuh et al., 1986). During annealing in a hydrogen atmosphere, bubbles of water steam were formed in the grain boundaries due to a reaction of hydrogen with the oxygen segregated at the boundaries. The total volume of the bubbles in low energy boundaries was smaller than that in high energy boundaries.

\section{THE EFFECT OF THE ELECTRONIC STRUCTURE ON THE BEHAVIOUR OF GRAIN BOUNDARIES IN METALS}

Most of the models dealing with the structure of grain boundaries are of a crystallographic nature and neglect electronic effects. However, several observations (Herrmann et al., 1976; Otawa and Ishida, 1977; Liebert and Thomas, 1984) suggest that in metals conduction electrons may be of significance for the behaviour of grain boundaries. The same conclusion has been reached theoretically by Seeger and Schottky (1959) who utilized a free electron gas model to calculate the energy of high angle boundaries. The fundamental idea of their calculation is that the packing density of the atoms in a grain boundary is lower than in the perfect lattice. This causes a larger average distance between the atoms in the boundary. Hence, a deficit of the charge density develops in the boundary region. The low positive charge density is screened by a local rearrangement of the conduction electrons. For the metals silver and copper, the calculated boundary energy agrees reasonably well with the experimental data. It was therefore concluded that the electronic contribution is responsible for the larger fraction of the grain boundary energy.

In order to assess the significance of the electronic structure on the energy of grain boundaries, systematic experiments on the low energy boundaries in pure $\mathrm{Cu}(99,99 \%), 67$ at. $\% \mathrm{Cu} 33$ at. $\% \mathrm{Ni}, 33$ at. $\% \mathrm{Cu} 67$ at. $\% \mathrm{Ni}$, pure $\mathrm{Ni}(99,99 \%)$, and 40 at.\% Ni 60 at.\% Co were carried out by applying the sphere rotation method (Maurer and Gleiter, 1985). As Cu-Ni forms solid solutions at all concentrations and as the lattice structure of all $\mathrm{Ni}-\mathrm{Cu}$ alloys is fcc with little variation $(2.53 \%)$ of the lattice constant, $\mathrm{Cu}-\mathrm{Ni}$-alloys provide a model system in which the electronic structure can be continuously varied from a transition metal to a noble metal without changing the 


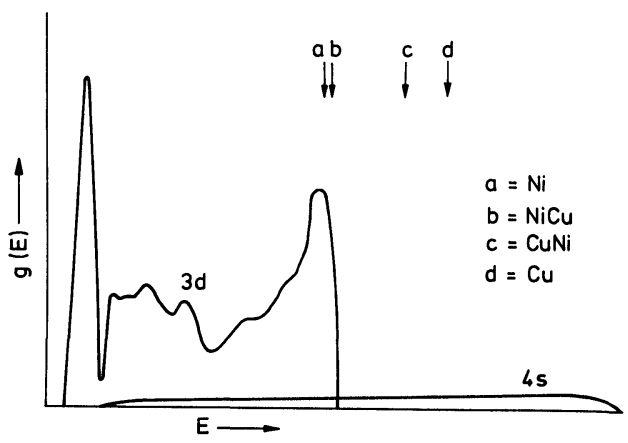

Figure 11 Density of states $g(E)$ in the $3 d$ and $4 s$ band as a function of the electron energy $E$. The arrows indicate the Fermi energy for (a) $\mathrm{Ni}$, (b) $\mathrm{Ni}-33$ at.\% $\mathrm{Cu}$, (c) Ni-66 at.\% Cu, (d) Cu (from Schulze, 1977).

crystallography. The $4 \mathrm{~s}$ and the $3 \mathrm{~d}$ bands of $\mathrm{Cu}$ and $\mathrm{Ni}$ have similar shapes. Both bands overlap (Figure 11). In alloys of $\mathrm{Cu}$ and $\mathrm{Ni}$, the $4 \mathrm{~s}$ electrons of $\mathrm{Cu}$ enter the empty energy levels of the $3 \mathrm{~d}$ band of $\mathrm{Ni}$. In other words, alloying $\mathrm{Cu}$ and $\mathrm{Ni}$ results primarily in a continuous shift of the Fermi energy from the level of pure Ni to the value of pure $\mathrm{Cu}$.

The experiments show that the number of low energy orientation relationships constantly decreases with increasing Ni contents. For pure $\mathrm{Ni}$ (above and below the Curie temperature) only $\Sigma 3$ boundaries were observed as low energy orientations. However, in pure $\mathrm{Cu}$, about 150 low energy orientations were found (Figure 12).

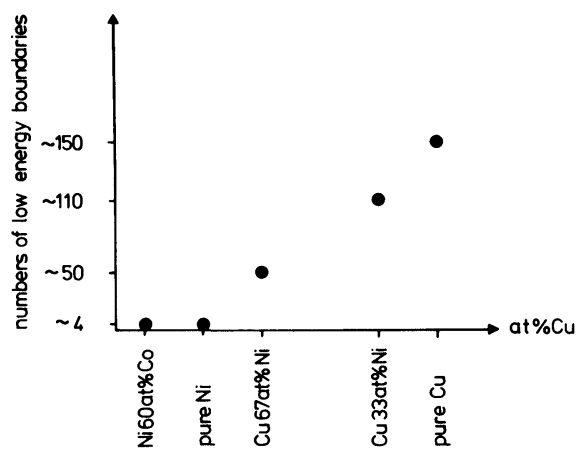

Figure 12 Decrease of the number of low energy boundaries with increasing Ni contents (from Maurer and Gleiter, 1986). 
No correlation between the $\Sigma$-value and the increasing number of special boundaries was noticed. As the lattice structure and the lattice constants of $\mathrm{Cu}-\mathrm{Ni}$-alloys remain unaffected when the chemical composition is varied, it is difficult to interpret the observed variation of the number of low energy boundaries in terms of a crystallographic model. The decreasing number of low energy orientations in $\mathrm{Ni}$ and $\mathrm{Ni}$-alloys may, however, be accounted for by electronic effects. In fact, the observations seem to suggest that the electronic structure controls the energy of grain boundaries to a large extent. This conclusion agrees with the results obtained for a $\mathrm{NiCo}$ alloy. $\mathrm{Ni}$ and $\mathrm{Co}$ (although chemically different) have comparable band structures. Indeed, little change of the boundary behaviour was noticed.

Two conclusions may be drawn from these observations: (i) Crystallographic parameters, such as coincidence site density, grain boundary periodicity, O-lattice site density etc. are not fully adequate to characterize the behaviour of boundaries. As the crystal lattices of all alloys investigated are identical, any type of crystallographic criterion would be the same for all alloys and, therefore, the same boundary behaviour would be expected. (ii) Computer simulations, based on pair-wise interaction potentials, do not seem to be entirely adequate to model the experimental observations reported, in particular the disappearance of all but one type of special grain boundaries $(\Sigma=3)$ in $\mathrm{Ni}$ and Ni-60 at \% Co. No matter which types of potentials are used to model the band structure change in $\mathrm{Cu}-\mathrm{Ni}$ alloys, grain boundary energy computations always indicate the existence of several low energy boundaries in pure $\mathrm{Ni}$, pure $\mathrm{Cu}$, and $\mathrm{Cu}-\mathrm{Ni}$ alloys. In order to account for this discrepancy between calculations and experimental observations, the equation for the boundary energy, $E$, in the computations should consist of, at least, two terms: one term, $F(n(\mathbf{r}))$, describing the electronic energy contribution of the system which only depends on the electron density and a structure dependent second term so that $E=F(n(\mathbf{r}))+1 / 2 \sum_{i j} V_{i j}$. Yet, the computations of the structure of grain boundaries published so far are exclusively based on the second term and result in predictions that are not in agreement with the experiments. This conclusion seems to be confirmed by measurements of the elastic constants of grain boundaries in metals (Korn et al., 1987). Young's modulus $E$ and the shear modulus $G$ of 
ultra-fine grained materials (Pd, $6 \mathrm{~nm}$ and $\mathrm{Mg}, 12 \mathrm{~nm}$ ) which consist of about 30 to $50 \mathrm{vol} \%$ of grain boundaries are comparable to those of conventional materials. The obtained results suggest that the interatomic potentials in grain boundaries differ from the lattice potentials.

\section{PHASE BOUNDARIES}

Erb et al. (1982) were the first to extend the sphere rotation method to the investigation of the boundary between two different materials in the example of a $\mathrm{NaCl}$-plate with $\mathrm{Ag}$ spheres. In a later study (Shirokoff and Erb, 1986), another three orientation relationships were found besides the two orientation types already known, due to an improved method. By means of the sphere-rotation-method, the orientation relationships observed in experiments with epitaxially grown layers (Matthews, 1965) were confirmed to be low energy orientations. However, the sphere-rotation-method offers better statistics than the expitaxial methods and thus, further low energy orientation relationships can be found.

By using the sphere-rotation method, Fecht and Gleiter (1985) investigated low energy interphase boundaries between different metal/ionic crystals. Their result confirmed Erb's finding that small

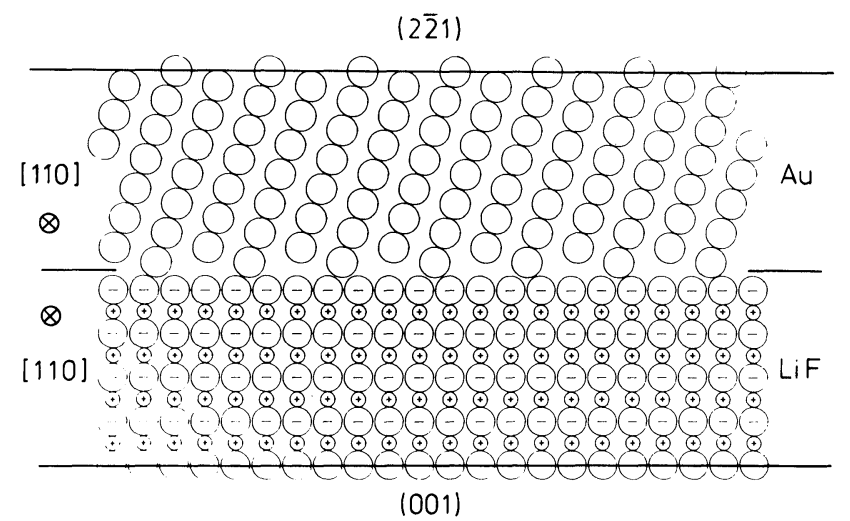

Figure 13 "Lock-in" between an $\mathrm{Au}$ - and a LiF-crystal when close packed [110]-rows of Au atoms "lock" into every third valley of the "(001)-surface" of the LiF crystal (from Fecht and Gleiter, 1985). 
lattice mismatch in the boundary is not the criterion controlling the energy of the boundaries as was expected according to the near CSL-model (Balluffi et al., 1982). In order to explain the experimental data obtained for a variety of different types of interphase boundaries, the lock-in-model was proposed. According to this model, a low energy interphase boundary is achieved if densely packed rows of atoms at the "surface" of the metal lock into the valleys between densely packed atom rows of the "surface" of the ionic crystal (Figure 13). The higher the packing density of the rows of atoms in lock-in configuration, the lower the energy of an interphase boundary. The correlated lattice strain is not discussed. It is expected that, during the rotation of the spheres, parameters such as temperature and the type of chemical bonding between sphere and plate are the crucial parameters for the interfacial energy.

In a paper discussing the low energy boundaries found in the $\mathrm{Ag} / \mathrm{Ni}$ system (Maurer and Fischmeister, 1987), it is shown that none of the existing geometrical models, such as the near CSL model or the lock-in-model, give an explanation of the observed orientation relationships. A comparison of these results with other sphere rotation studies reveals considerable similarity between all

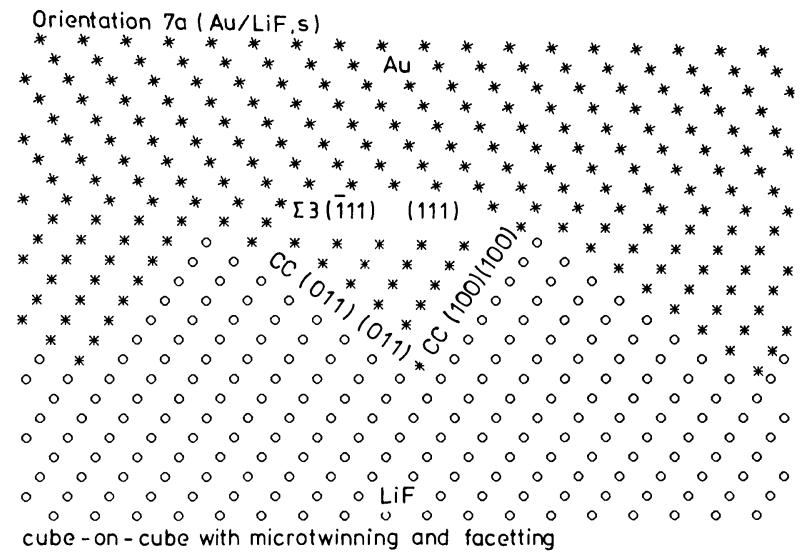

Figure 14 Example for the assumed reduction: micro-twinning and facetting of the $(11 \overline{1})_{\mathrm{Au}}\left\|(111)_{\mathrm{LiF}},[01 \overline{1}]_{\mathrm{Au}}\right\|[01 \overline{1}]_{\mathrm{LiF}}$ boundary to form cube-on-cube boundaries (from Maurer and Fischmeister, 1987a). 
Table 1 Crystallographic characterization of the basic set of boundary orientation types

\begin{tabular}{ccc}
\hline Nr. & Parallel planes & Parallel directions* \\
\hline 1. & cube-on-cube & \\
2. & $(111)_{s} \|(100)_{p}$ & {$[01 \overline{1}]_{s} \|[01 \overline{1}]_{p}$} \\
3. & $(211)_{s} \|(100)_{p}$ & {$[011]_{s} \|[011]_{p}$} \\
4. & $(111)_{s} \|(011)_{p}$ & {$[011]_{s} \|[01 \overline{1}]_{p}$} \\
5. & $(211)_{s} \|(011)_{p}$ & {$[01 \overline{1}]_{s} \|[01 \overline{1}]_{p}$} \\
6. & $(011)_{s} \|(100)_{p}$ & {$[01 \overline{1}]_{s} \|[01 \overline{1}]_{p}$} \\
7. & $(100)_{s} \|(100)_{p}$ & {$[01 \overline{1}]_{s} \|[010]_{p}$} \\
8. & $(111)_{s} \|(100)_{p}$ & {$[011]_{s} \|[010]_{p}$} \\
9. & $(211)_{s} \|(100)_{p}$ & {$[011]_{s} \|[010]_{p}$} \\
10. & $(111)_{s} \|(011)_{p}$ & {$[01 \overline{1}]_{s} \|[100]_{p}$} \\
\hline
\end{tabular}

* The subscripts $s$ and $p$ refer to planes and directions in the lattices of the spheres and plates.

systems studied so far, including metals on oxides and on alkali halides. Assuming that the boundaries across the necks can both facet and form occluded $\Sigma 3$ twins (Figure 14), it is possible to reduce all 33 orientation types to 10 "elementary" boundary types (Table 1). In almost all systems, cube-on-cube is the most dominant orientation.

All remaining observed boundary types can then be described as boundaries in which a twinning plane of one material is parallel to a low index plane of the other material. An analysis of the preferred "elementary" boundaries shows that they could be understood as coincidence boundaries with a considerably extended tolerance radius. It is suggested that such "extended coincidence" might not be unreasonable for pairs of materials with weak interphase bonding.

Latest studies show that the orientation distribution of small silver spheres annealed on amorphous silica substrates reveals two cusps in the interfacial energy vs. misorientation curve (Shirokoff and Erb, 1987). These cusps correspond to orientations for which close-packed and second close-packed planes lie parallel to the silica surface. They are the same preferred orientations as previously reported for silver films as grown from the vapour phase on glass substrates. By investigating the $\mathrm{Ag} / \mathrm{SiO}_{\mathrm{x}} / \mathrm{Si}$ interfacial system, three orientation relationships of low energy were observed (Shirokoff et al., 1987). Two of these are identical to the cusps found for the 
$\mathrm{Ag} /$ bulk silica system. The third orientation relationship is the $\mathrm{Ag} / \mathrm{Si}$ epitaxy orientation which is present even when an oxide layer of about $180 \AA$ in thickness separates the two phases. It appears that long range ordering effects in this system exist over distances of at least $180 \AA$.

\section{References}

Balluffi, R. W., Brokman, A., King, A. H. (1982) CSL/DSC Lattice model for general crystal-crystal boundaries and their line defects. Acta Metallurgica, 30, 1453-1470.

Bunge, J., Gleiter, H. (1987) Current research supported by DFG.

Erb, U., Gleiter, H. (1979). The effect of temperature on the energy and structure of grain boundaries. Scripta Metallurgica, 13, 61-64.

Erb, U., Abel, W., Gleiter, H. (1982) The significance of atomic matching for the structure of interphase boundaries. Scripta Metallurgica, 16, 1317-1319.

Erb, U., Gleiter, H., Schwitzgebel, G. (1982) The effect of boundary structure (energy) on interfacial corrosion. Acta Metallurgica, 30, 1377-1380.

Fecht, H. J., Gleiter, H. (1985) A lock-in model for the atomic structure of interphase boundaries between metals and ionic crystals. Acta Metallurgica, 33, 557-562.

Goodhew, P. J., Allen, R. M. (1977) Comments on. "Low energy grain boundaries in metals". Scripta Metallurgica, 11, 37-39.

Goodhew, P. J. (1980) The relationship between grain boundary structure and energy. In Grain Boundary Structure and Kinetics, edited by R. W. Balluffi, ASM Seminar 1979, American Society for Metals, Metals Park, OH, pp. 155-179.

Herrmann, G., Gleiter, H., Bäro, G. (1976) Investigation of low energy grain boundaries in metals by a sintering technique. Acta Metallurgica, 24, 353-359.

Korn, D., Morsch, A., Birringer, R., Arnold, W., Gleiter, H. (1987) Measurements of the elastic constants, the specific heat, and the internal friction of grain boundaries by means of ultra-fine grained materials. Journal de Physique (Paris) Colloque, in press.

Liebert, M., Thomas, L. (1984) Optische Eigenschaften von Korngrenzen. Zeitschrift für Metallkunde, 75, 273-297.

Lojkowski, W., Gleiter, H. (1985) Low energy grain boundaries in silver. Journal de Physique (Paris), Colloque C4, 46, C4-89-C4-94.

Lojkowski, W., Gleiter, H., Maurer, R. (1987) Low energy grain boundaries in silver and copper close to the melting point. Acta Metallurgica, in press.

Matthews, J. W. (1965) Growth of Face Centered Cubic Metals on Sodium Chloride Substrates. Journal of Vacuum Science and Technology, 3, 3, 133-145.

Maurer, R., Erb, U., Gleiter, H. (1984) Intercrystalline corrosion: Factors controlling the enhanced corrosion near grain boundaries. Materials Science and Engineering, 63, L13-L15.

Maurer, R., Gleiter, H. (1985) The effect of the electronic structure on the behaviour of grain boundaries in metals. Scripta Metallurgica, 19, 1009-1012.

Maurer, R., Gleiter, H. (1986) Investigations of grain boundary properties by means of texture measurements. In Experimental Techniques of Texture Analysis, ed. by H. J. Bunge, DGM Informationsgesellschaft Verlag, pp. 343-357.

Maurer, R. (1987) Improved technique for the determination of low energy 
boundaries by the rotating-sphere-on-a-plate method. Results for grain boundaries in the Cu/Ni system. Acta Metallurgica, in press.

Maurer, R., Fischmeister, H. F. (1987a) Heterophase boundaries in the Silver-Nickel system. Journal de Physique (Paris) Colloque, in press.

Maurer, R., Fischmeister, H. F. (1987b) Low energy orientation relationships of $\mathrm{Ag}-\mathrm{Ni}$ interfaces examined by the sphere-rotation method. To be published.

Meiser, H., Gleiter, H. (1980) The effect of hydrostatic pressure on the energy of grain boundaries-structural transformations. Scripta Metallurgica, 14, 95-99.

Mykura, H. (1980) Appendix: A checklist of cubic coincidence site lattice relations. In Grain-Boundary Structure and Kinetics, ASM Seminar 1979, American Society for Metals, Metals Park, Ohio, pp. 445-456.

Ozawa, T., Ishida, Y. (1977) Mössbauer effect of ${ }^{119 m}$ Sn segregation at the grain boundary of iron. Scripta Metallurgica, 11, 835-838.

Roy, A., Erb, U., Gleiter, H. (1982) Grain boundary embrittlement as a function of boundary structure (energy). Acta Metallurgica, 30, 1847-1850.

Sautter, H., Gleiter, H., Bäro, G. (1977) The effect of solute atoms on the energy and structure of grain boundaries. Acta Metallurgica, 25, 467-473.

Schuh, Th., Hahn, H., Gleiter, H. (1986) Grain boundary structure and boundary segregation in Ag-O alloy. Materials Science and Engineering, 84, 191-193.

Schulze, G. (1974) Metallphysik, 2. ed., p. 312. Akademie-Verlag, Berlin.

Seeger, A., Schottky, G. (1959). Die Energie und der elektrische Widerstand von Großwinkelkorngrenzen in Metallen. Acta Metallurgica, 7, 495-503.

Shewmon, P. G. (1966) Energy and structure of grain boundaries. In Recrystallization, Grain Growth and Textures, ASM Seminar, American Society for Metals, Metals Park, Ohio, pp. 196-198.

Shirokoff, J., Erb, U. (1986) Detection of the complete set of preferred orientations of silver on sodium chloride. Scripta Metallurgica, 20, 1607-1611.

Shirokoff, J., Erb, U. (1987) Measurement of preferred orientations in the silver-silica interface. Thin Solid Films, 151, 65-70.

Shirokoff, J., Hui, K. C., Erb, U. (1987) The study of long range forces in epitaxy systems: The Ag/Si interface. Journal de Physique (Paris) Colloque, in press.

Sutton, A. P., Balluffi, R. W. (1987) On geometric criteria for low interfacial energy. Acta Metallurgica, in press.

Wirth, R., Gleiter, H. (1981) Is discontinuous (cellular) precipitation an effect of a structural transformation in the migrating phase boundary? Acta Metallurgica, 29, 1825-1830. 